\title{
Retrato da Dor: Um Caminho para Entender o Sofrimento do Indivíduo
}

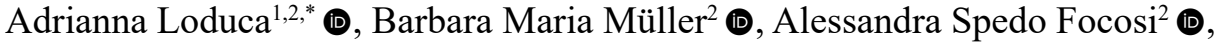 \\ Claudio Samuelian² (D, \& Lin Tchia Yeng ${ }^{2}$ (D) \\ ${ }^{1}$ Faculdade de Ciências Humanas e da Saúde da Pontifícia Universidade Católica de São Paulo, São Paulo, SP, Brasil \\ ${ }^{2}$ Centro de Dor do Hospital das Clínicas da Faculdade de Medicina da Universidade de São Paulo, São Paulo, SP, Brasil
}

\begin{abstract}
RESUMO - Este estudo tem por objetivo verificar o sofrimento associado às dores crônicas, por meio do instrumento projetivo "Retrato da Dor". Trata-se de um estudo descritivo, realizado com 126 pacientes com dores crônicas de diversas etiologias. As respostas foram analisadas de acordo com o método de análise de conteúdo de Bardin e testes estatísticos. Foram encontrados pacientes: que acreditam que nenhum sofrimento se compara com a sua dor (34,9\%); que comparam sua dor a outras dores ou doenças já experienciadas (16,6\%); e os que comparam a dor a situações de sofrimento emocional $(48,4 \%)$. Este estudo evidenciou que os aspectos emocionais desempenham um papel importante na maneira como o indivíduo irá interpretar e utilizar recursos próprios para lidar com suas dores.
\end{abstract}

PALAVRAS-CHAVE: dor crônica, narração, desenho

\section{Pain Portrait: A Way to Understand Individual Suffering}

\begin{abstract}
This study aims to verify the suffering associated with chronic pain through the projective instrument "Pain Portrait". It is a descriptive study carried out with 126 patients with chronic pain of different etiologies. Responses were analyzed according to the Bardin content analysis method and statistical tests. Patients were found: who believe that no suffering compares to their pain (34.9\%); Who compare their pain to other pains or diseases already experienced (16.6\%); And those who compare pain to situations of emotional distress (48.4\%). This study showed that emotional aspects play an important role in how the individual will interpret and use his or her own resources to deal with their pain.
\end{abstract}

KEYWORDS: Chronic Pain, narration, design

A dor tem sido compreendida pelas pessoas como um sinal ou sintoma de que algo no corpo não está funcionando corretamente; ou seja, é considerada um sinal de alerta. Porém, quando se prolonga por mais de três meses, ela se torna crônica e deixa de ser um sintoma, passando a ser uma doença que pode ter diferentes diagnósticos e requer condutas distintas, dependendo da definição diagnóstica (Merskey, 1991).

A referência ao conceito de dor é imediatamente associada a sofrimento. Desde 1979 até meados de outubro de 2016, a IASP (International Association for the Study of Pain) adotava a definição de dor crônica como: "uma experiência sensorial e emocional desagradável associada a lesão tecidual real ou potencial ou descrita em termos de tal lesão" (Merskey, 1991, p.157). Essa definição excluía características importantes e clinicamente relevantes, como os aspectos cognitivos e sociais, e caracterizava a experiência simplesmente como "desagradável". Porém, nem toda experiência desagradável causa dor. Esses e outros contrapontos conduziram a IASP, em 2019, a criar uma força-tarefa para rever a definição da dor crônica. E, em 2020, a definição de 1979 foi ratificada e expandida pela adição de seis notas e da etimologia da palavra dor: (1) experiência pessoal que é influenciada em vários graus por fatores biológicos, psicológicos e sociais; (2) não pode ser inferida apenas da atividade dos neurônios sensoriais; (3) conceito aprendido; (4) o relato de uma pessoa de uma experiência como dor deve ser respeitado; (5) pode ter efeitos adversos na função e no bem-estar social e psicológico; (6) etimologia: inglês médio, do anglo-francês

\footnotetext{
*E-mail: contato.tapsi@gmail.com

- Submetido: 30/11/2017; Aceito: 12/01/2021.
} 
peine (dor, sofrimento), do latim poena (pena, punição), por sua vez do grego poine (pagamento, pena, recompensa) (Raja et al., 2020).

Considerando as pessoas que convivem com quadros de dor por um período prolongado, ou seja, aquelas que sofrem com dores crônicas, é inegável o impacto no dia a dia. Estudos na área revelam que o quadro álgico crônico provoca alterações na vida de uma pessoa em vários âmbitos: físico, psicológico (cognitivo, emocional e comportamental), social e econômico, que são, muitas vezes, difíceis de ajustar. No que se refere ao impacto físico, a dor crônica, com frequência, pode reduzir a mobilidade, provocar alterações no sono e fadiga. Quanto à interferência psicológica, pode causar ansiedade, depressão, raiva e pensamentos catastróficos e, nos aspectos socioeconômicos, pode ocasionar afastamento do trabalho, diminuição do lazer e dos rendimentos, dependência de cuidados alheios e conflitos nas relações interpessoais (Wall \& Melzack, 1999; Linton, 2000; McBeth et al., 2001; Buer \& Linton, 2002, Jakobsson et al., 2003, Keefe et al., 2004, Manek \& Macgregor, 2005, Kreling, Cruz \& Pimenta, 2006, Menzel, 2007). Esses impactos que comprometem a vida dos sofredores de forma global resultam em incertezas, medos, preocupações e sensação de incapacidade (Yeng \& Teixeira, 2004; Loduca \& Samuelian, 2009; Szumita, Szumita, \& Just, 2010).

No Brasil, existem poucos estudos epidemiológicos. Estima-se que a prevalência de dor crônica é de 30\% a 50\%; acomete 1 a cada 5 adultos; aumenta com o avanço da idade e tende a atingir mais mulheres do que homens (Cabral et al., 2014; Sá et al., \& Jansen, 2012). Os indivíduos acometidos por dores crônicas alegam que elas comprometem suas atividades habituais e mais de $3 / 4$ deles consideram que limitam as atividades profissionais e de lazer, assim como interferem nas relações sociais e familiares (Ferreira, Siqueira, \& Teixeira, 2008). Além disso, as dores crônicas predominam na faixa dos 30 aos 50 anos de idade, o que ameaça a produtividade da população brasileira. Muitas pessoas com dores crônicas não retornam ao trabalho. Constatou-se que menos de $50 \%$ dos pacientes com dor lombar retornavam à atividade laboral, após 6 meses de afastamento (Yeng \& Teixeira, 2004; Ferreira et al., 2008).

Mundialmente, estudos sinalizam que a incidência de dor crônica na população adulta é de $20 \%$ e costumam ter a duração média de 7 anos. Todo ano, 1 em cada 10 adultos são diagnosticados (Goldberg \& McGee, 2011).

A busca por novas estratégias de tratamento e prevenção de dores crônicas tem se constituído em uma das prioridades na saúde pública mundial, despertando a necessidade de se expandirem os estudos, diagnósticos e tratamentos (Goldberg \& McGee, 2011). Assim, as dificuldades para manejo ou remissão do quadro álgico crônico evidenciaram para a IASP a importância de os Centros de Dor compreenderem e tratarem os quadros álgicos crônicos sob a perspectiva biopsicossocial, o que requer o estabelecimento de tratamentos multi ou interdisciplinares. Muito embora os pacientes procurem essa assistência, nem sempre eles se mostram disponíveis para realizar as condutas indicadas pela equipe; ou seja, eles querem a remissão do quadro doloroso e esperam que a ingestão de remédios ou procedimentos clínicos, em um curto prazo, possam eliminar o desconforto álgico (Loduca \& Samuelian, 2009).

Ao longo da história, inúmeras teorias foram desenvolvidas na tentativa de explicar os mecanismos de ação da dor, já que ela aparece como uma experiência singular. Há 50 anos, Melzack e Wall (1967) desenvolveram a Teoria do Controle da Comporta, com o objetivo de explicar como a medula espinal e o cérebro agiam para a manutenção da dor crônica, abrindo espaço para que outras pesquisas fossem desenvolvidas (Flor et al., 1998). Esses estudos levaram ao desenvolvimento da Teoria da Neuromatriz da Dor, segundo a qual a dor não é um fenômeno puramente sensorial, reforçando a necessidade de avaliar e tratar a pessoa como um todo e não apenas a dor. A Teoria da Neuromatriz da Dor ratificou que os aspectos emocionais desempenham um papel importante na maneira como o indivíduo irá interpretar e utilizar recursos próprios para lidar com suas dores, portanto, não se deve negligenciar seu discurso (Melzack, 2001; Melzack 2005). Nesse sentido, a análise da narrativa de cada paciente deve ser considerada como um elemento fundamental a ser abordado pelas equipes de saúde nos centros para tratamento de dores crônicas (Loduca, 2007).

A literatura científica tem evidenciado estudos que explicam que a cultura pode interferir na experiência de dor em diversos aspectos: nas respostas individuais (sensações, emoções, crenças e enfrentamento), nas relações interpessoais e nas expectativas sociais, sobretudo no significado atribuído a essa vivência, tanto do ponto de vista do paciente quanto do profissional. Entende-se que o significado associado à dor e ao sofrimento correspondente é fruto da influência de ideologias sobre saúde, qualidade de vida e status socioeconômico, e o sentido dado irá mediar as relações interpessoais entre o paciente e a equipe de saúde que o assiste (Campbell et al., 2009, Shavers; Bakos, \& Sheppard, 2010). Logo, a sobrecarga que uma doença pode provocar na vida de uma pessoa é uma experiência pessoal normalmente comunicada pela expressão verbal e comportamental que, muitas vezes, denuncia a escassez de palavras ou gestos que possam retratar com fidedignidade a dor vivenciada. Percebe-se que a expressão de dor e sofrimento é constituída pela somatória de fatores físicos, emocionais e sociais, portanto, não pode ser traduzida apenas pela quantificação da frequência e da intensidade do quadro álgico ou pela busca por palavras que qualifiquem o sofrimento vivenciado (McWhinney, 1981).

É importante ajudar os pacientes a encontrarem os meios que melhor possam expressar aquilo que estão sentindo. Assim, o desenho aparece como um recurso que tem sido utilizado na área da arte para expressar emoções, sentimentos e sensações que não encontram eco ou total ressonância através da linguagem verbal ou comportamental. 
Um exemplo de como podemos expressar as angústias de modo diferente são as obras da pintora mexicana Frida Khalo, que, particularmente, comunicou em vários de seus quadros o impacto de seu convívio com distintas condições de sofrimento, incluindo dores crônicas.

A carência de instrumentos que auxiliem na compreensão do impacto e das repercussões da dor na vida dos pacientes, aliada à demanda dos profissionais em buscar novos caminhos que transponham a validação de instrumentos internacionais, evidenciam, assim como referido na literatura mundial, a necessidade de desenvolver novos recursos que venham responder os questionamentos das equipes e a realidade de nossa população.

Deve-se lembrar que estudos revelam diferenças entre grupos étnicos e culturais na maneira de se expressarem e darem sentido à experiência angustiante de dor e na capacidade de enfrentamento e manejo dessa condição (Goldman et al., 2002; Urnes et al., 2006). Dentro dessa perspectiva, Loduca (1998) desenvolveu um instrumento projetivo, que denominou como Retrato da Dor. Na época, a autora percebeu que pacientes que tinham o mesmo diagnóstico de dor não necessariamente atribuíam o mesmo sentido às suas dores e, como não existiam exames que as retratassem, se questionou se não seria interessante solicitar que o paciente tentasse concretizar a sua experiência através da representação gráfica de seu desconforto físico. $\mathrm{Na}$ sequência, realizava-se um inquérito para ampliar a compreensão do sentido da dor na vida do paciente e, assim, o Retrato da Dor permitia ao paciente expressar seu sofrimento, ou seja, abordava-se a dor para além do sofrimento físico, procurando compreendê-la dentro da biografia de cada um (Loduca, 2007; Loduca et al., 2014). Percebeu-se que esse recurso amenizava os mecanismos de racionalização do indivíduo, permitindo a obtenção de informações importantes e, ainda, o reconhecimento da influência de fatores psicológicos na percepção da dor (Loduca \& Samuelian, 2003). Por vezes, o inquérito mobilizava emoções no paciente que o fazia perceber ou reconhecer a demanda de assistência psicológica (Loduca \& Samuelian, 2003; Loduca \& Samuelian, 2009) e, neste sentido, tornou-se um recurso que poderia ser útil tanto nos consultórios quanto nos atendimentos ambulatoriais ou nas enfermarias dos hospitais, favorecendo a adesão ao tratamento psicológico.

Enquanto no Brasil estavam sendo valorizados a narrativa e o sentido que cada paciente atribuía às suas dores, também por volta de 2008 surgiu um movimento denominado PainSTORY, desenvolvido em países europeus que sinalizaram a preocupação de dar atenção para a narrativa e a expressão gráfica da dor dos pacientes. O estudo foi realizado, ao longo de um ano, com 294 pacientes de 13 países europeus, com o objetivo de verificar o impacto da dor crônica na vida deles. Os resultados do PainSTORY revelaram que a dor assumia diferentes significados na vida dos doentes, reforçando a importância de valorizar o discurso de cada um e a riqueza de utilizar outras formas de expressão da dor, como a produção gráfica (O’Brien, \& Breivik, 2012).

Observam-se na literatura mundial poucos relatos do uso de instrumentos projetivos nos estudos psicológicos sobre avaliação e tratamento de dores crônicas. Dentre os relatos encontrados, utilizam-se principalmente instrumentos projetivos já existentes na área de Psicologia, como o Minnesota Multiphasic Personality Inventory (MMPI), o Rorchasch, o Teste de Apercepção Temática (TAT) e o Desenho da Figura Humana (DFH) (Panek, Skowronski, \& Wagner, 2002; Villemor-Amaral \& Pasqualini-Casado, 2006).

Existe uma lacuna no que se refere ao desenvolvimento de novos instrumentos projetivos para facilitar a avaliação de pessoas com dores crônicas. Essa constatação mostra a premência de serem utilizados outros recursos na área da dor que permitam que o paciente possa expressar seu sofrimento de forma mais livre e criativa. As técnicas projetivas requerem que o indivíduo utilize características de sua personalidade, necessidades e experiências de vida para interpretar estímulos ambíguos, o que diminui a ação de mecanismos de defesa e permite o acesso a conteúdo não acessível à consciência da pessoa testada (Farias Oliveira Nunes et al.,2008).

O "Retrato da Dor" tem se mostrado para os psicólogos uma ferramenta que auxilia no trabalho de intervenção (Loduca et al., 2014). Esse estudo faz parte de um projeto que tem sido desenvolvido desde 2014, ano no qual foi publicado o artigo supracitado, cujas categorias de desenhos foram apresentadas à comunidade científica e que foi aprovado no Comitê de Ética da Pontifícia Universidade Católica de São Paulo, sob o n ${ }^{\circ}$ 25463613.1.0000.5482. Também foram realizadas duas pesquisas na modalidade Iniciação Científica e publicado um e-book denominado "Retrato de Dores" (Loduca, Müller, \& Samuelian, 2018). Nesse momento, estamos atentos à questão do inquérito, na qual verificamos se o paciente associa alguma vivência anterior com a dor crônica por meio do instrumento projetivo "Retrato da Dor" (Loduca, 1998), sendo este o objetivo da presente pesquisa.

\section{MÉTODO}

Estudo descritivo realizado com 126 pacientes com dores crônicas de diversas etiologias, encaminhados pelo grupo de Dor do Instituto de Ortopedia e Traumatologia do Hospital das Clínicas da Faculdade de Medicina da
Universidade de São Paulo (HC FMUSP), entre agosto de 2016 e julho de 2017.

Todos os participantes acima dos 18 anos e contatáveis por telefone eram candidatos a participar do estudo, 
entretanto, foram excluídos os que apresentavam alto grau de comprometimento cognitivo, comorbidades psiquiátricas graves ou que se recusaram a participar. Os pacientes que colaboraram nessa pesquisa assinaram o termo de consentimento livre e esclarecido, conforme as diretrizes e normas regulamentadoras de pesquisas envolvendo seres humanos da Resolução nº66 de 12 de dezembro de 2012 do Conselho Nacional de Saúde. Foram avaliados, no início do tratamento, segundo três grupos de variáveis: (1) sociodemográficas: sexo, idade, estado civil, religião, escolaridade; (2) relacionadas à dor: tempo de convívio com a dor e intensidade de dor (escala numérica - o profissional questiona o paciente sobre a nota que ele atribui à sua dor de 0 a 10 , sendo que " 0 " significa não ter dor e " 10 ", uma dor insuportável); (3) as respostas dadas à última questão do inquérito do instrumento "Retrato da Dor" (Loduca,
1998; Loduca et al., 2014). Esta última variável foi avaliada a partir da questão "Teve algum momento na sua vida que tivesse sido igual ou pior do que esta dor? Não precisa estar relacionado com doença, pode ser qualquer coisa que se assemelhe ao seu sofrimento atual".

As respostas dadas ao inquérito do instrumento "Retrato da Dor" foram analisadas de acordo com o método de análise de conteúdo (Bardin, 2004). Os grupos de variáveis foram correlacionados entre si por meio do software estatístico SPSS (Statistical Package for the Social Sciences). Foram calculadas as frequências de cada resposta por meio dos testes Qui-quadrado e Mann-Whitney, para investigar a existência de correlações entre as variáveis e o teste ANOVA e verificar a variação entre as medias dos grupos. O nível de significância adotado nesta pesquisa foi de 0,05 .

\section{RESULTADOS}

A amostra foi composta 126 pacientes. Desses, 50,8\% são homens, cujas médias de idade, intensidade de dor e tempo de convívio com a dor foram de 47,7 anos ( $\mathrm{DP}=14,12)$, $5,83(\mathrm{DP}=2,46)$ e 66,5 meses $(\mathrm{DP}=71,23)$, respectivamente.

Ainda em relação à amostra, foram examinadas variáveis como religião, nível de escolaridade e estado civil. As religiões predominantes neste estudo foram católica $(50 \%)$ e evangélica $(28,7 \%)$. Quanto ao nível de escolaridade, $39,6 \%$ têm ensino médio e $24,5 \%$ cursaram ensino fundamental I ( $1^{\circ}$ ao $5^{\circ}$ anos). Em relação ao estado civil, $63,2 \%$ estavam casados.

A partir da análise de conteúdo realizada em relação a última questão do inquérito do "Retrato da Dor", foram identificadas algumas categorias que comparam a dor crônica com outras situações de sofrimento vividas. Cerca de $34,9 \%$ das pessoas falaram que nada se comparava à dor sentida, sendo que, destas, $63,6 \%$ eram homens $(22,2 \%$ da amostra total). Outros $6,3 \%$ dos participantes da pesquisa relataram que já sentiram sofrimento semelhante a essa dor em outras situações, não havendo diferença em relação ao sexo, visto que $50 \%$ dos homens e das mulheres $(3,15 \%$ da amostra total) escolheram essa opção. A maioria dos pacientes, 58,7\%, no entanto, afirmou que essa dor atual era o pior evento de suas vidas e, desses, 56,7\% foram representados por mulheres (33,3\% do total da amostra).

$\mathrm{Na}$ Figura 1 podemos ver a distribuição das respostas pelo sexo do participante. Dentre as respostas de cunho emocional, que totalizam $48,4 \%$, percebe-se que a maior parte das mulheres assemelha a dor a um sofrimento de perda de um ente querido (luto - 18,3\%), dificuldade

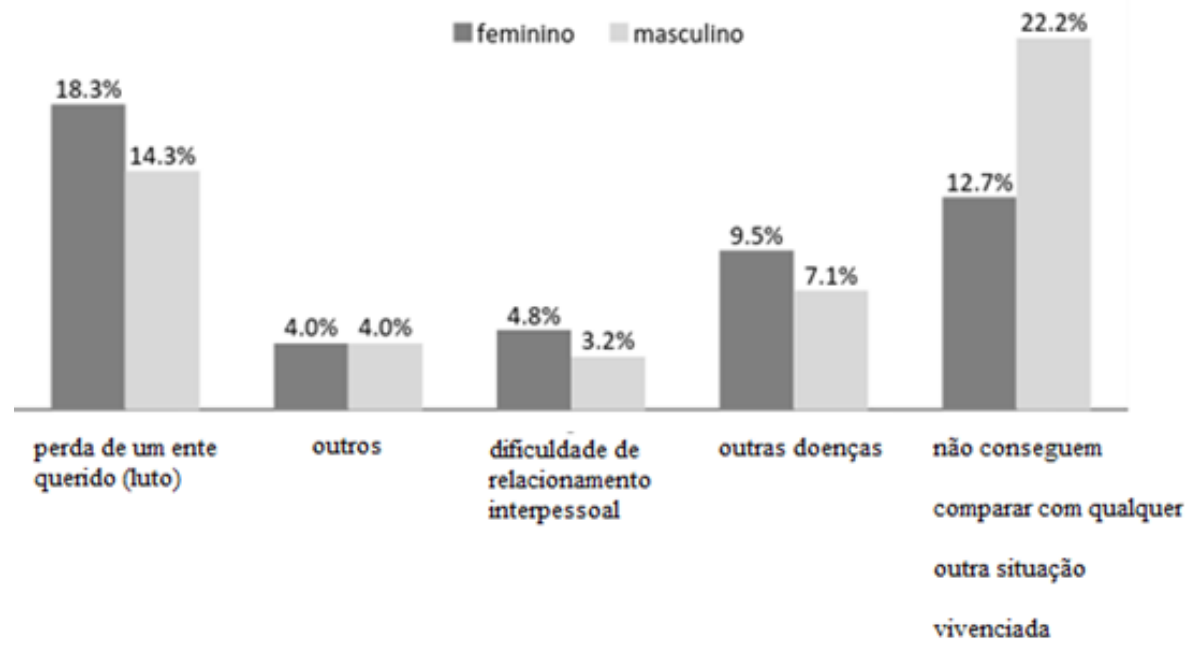

Figura 1. Distribuição das respostas de homens e mulheres frente ao sofrimento semelhante à dor crônica 
de relacionamento interpessoal $(4,8 \%)$ e outros tipos de respostas que apareceram igualmente tanto nos homens como nas mulheres (4\%). Pensando em pessoas que comparam o sofrimento físico causado pela dor crônica com outras dores ou outras doenças $(16,6 \%)$, novamente percebemos um predomínio da população feminina (9,5\%). Algumas pessoas não conseguem comparar o sofrimento que sentem com a dor crônica com qualquer outra situação vivenciada anteriormente. Dentre estas, destacam-se os homens que correspondem a 22,2\% da amostra.

Ao efetuar os testes de Qui-quadrado e Mann-Whitney (Tabela 1), não foram observadas correlações entre as variáveis estudadas e, ao realizar os testes ANOVA e Kruskal-Wallis (Tabela 2), não se identificou diferença significativa entre as médias.

Tabela 1

Teste de Qui-quadrado e Mann-Whitney

\begin{tabular}{lcc}
\hline & Qui-Quadrado & Mann-Whitney \\
\hline Momento igual ou pior X Sexo & $0,101^{\mathrm{a}}$ & 0,911 \\
Momento igual ou pior X Religião & $0,473^{\mathrm{a}}$ & 0,409 \\
Momento igual ou pior X Escolaridade & $0,353^{\mathrm{a}}$ & 0,531 \\
Momento igual ou pior X Estado Civil & $0,206^{\mathrm{a}}$ & 0,951 \\
\hline
\end{tabular}

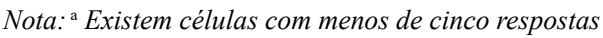

Tabela 2

Teste ANOVA e Kruskal-Wallis

\begin{tabular}{lcc}
\hline & ANOVA & Kruskal-Wallis \\
\hline Momento igual ou pior X VAS & 0,261 & 0,2095 \\
Momento igual ou pior X Idade & 0,237 & 0,1367 \\
\hline
\end{tabular}

\section{DISCUSSÃO}

Nessa pesquisa, foi observado um número muito próximo de homens e mulheres, o que difere dos dados da literatura nacional, que afirmam que a dor é mais frequente na população feminina (Kreling, Cruz \& Pimenta, 2006).

Em nosso estudo, a idade mínima observada foi de 20 anos e a máxima foi de 85 anos. Sendo assim, a média de idade coincide com a de outros estudos nacionais (Yeng \& Teixeira, 2004; Ferreira et al., 2008; Cabral et al., 2014; Sá et al., 2008; Moraes Vieira et al., 2012), demonstrando que a população mais afetada pela dor crônica é a que está em idade economicamente ativa.

Com relação à intensidade média de dor, obtivemos o valor de 5,83. O resultado corrobora com a pesquisa realizada por Cabral et.al (2014), segundo a qual a intensidade média de dor na cidade de São Paulo foi de 5,9 numa escala de 0 a 10.

Não foram encontrados estudos similares ao realizado e existem poucos estudos que utilizam instrumentos projetivos para avaliação e tratamento de pacientes com dores crônicas (Ocampo et al., 2001).

A dor tem um caráter subjetivo e peculiar, o que dificulta a generalização de informações e a identificação de perfis de pacientes (Loduca et al., 2014), tal como observado também na pesquisa atual.

Neste estudo, $48,4 \%$ dos participantes relacionaram aspectos emocionais vividos anteriormente ao sofrimento atual, o que reforça a importância da narrativa, pois é o que embasa a interpretação e a busca por recursos próprios para lidar com a dor crônica. Loduca, em 2007, já afirmava que a análise da narrativa deveria ser mais um elemento utilizado pelos profissionais da área da saúde que atendem pacientes com dores crônicas, para avaliar e auxiliar na definição de condutas terapêuticas.

Conforme visto na seção anterior, algumas das respostas dadas à questão sobre as situações de sofrimento iguais ou piores à vivida, em decorrência do quadro álgico, foram categorizadas em luto, dificuldades de relacionamento interpessoal e outras. De acordo com a literatura, principalmente quando o paciente se depara com situações similares àquelas anteriormente mencionadas, é importante que ele receba assistência psicológica, evitando, assim, que essas variáveis atuem na manutenção, no agravamento ou em prejuízo do tratamento proposto para o manejo da dor (Loduca et al., 2014). 
Nesse sentido, o papel da Psicologia vem ganhando destaque nos últimos anos na área da dor, assim como em equipes de saúde multi e interdisciplinares, não só pela recomendação da IASP para uma abordagem biopsicossocial, mas também pelos resultados que apresentam em suas intervenções e pela multidimensionalidade dos processos de adoecimento (Garcia Capitão, Scortegagna \& Baptista, 2005).

Outro grupo de pacientes que nos chamou a atenção foi daqueles que afirmaram que a dor era a pior situação de sofrimento de suas vidas, pois todos os indivíduos passaram por situações diversas e dificuldades ao longo da vida. Sendo assim, é necessário empoderá-los para que consigam encontrar recursos que os ajudem a lidar com a dor, de modo a conseguir enxergar as suas potencialidades e desenvolvê-las, em vez de manterem-se focados nas suas limitações, tal como afirmam Taddeo et al. (2012).
A vivência de empoderamento psicológico frente a situações de carência ou ruptura permite aos pacientes reconhecerem não apenas recursos e possibilidades pessoais ou coletivas, mas também sua capacidade de sair de uma posição de impotência e resignação, transformando-se em sujeitos ativos que lutam para si, por si e com mais autonomia e autodeterminação, tomando a direção da vida nas próprias mãos (Taddeo et al., 2012).

Concluindo, nossos resultados demonstram que a utilização do instrumento "Retrato da Dor" favorece a expressão do paciente e de seu sofrimento, dando significado ao processo doloroso e enfatizando as influências dos aspectos psicológicos (Loduca et al., 2014). Esta pesquisa nos instiga a fazer novos estudos para ampliar e aprofundar os potenciais do instrumento projetivo "Retrato da Dor".

\section{REFERÊNCIAS}

Bardin, L. (2004). Análise de conteúdo. Edições 70.

Buer, N., \& Linton, S. J. (2002). Fear-avoidance beliefs and catastrophizing: occurrence and risk factor in back pain and ADL in the general population. Pain, 99(3), 485-491. https:// doi.org/10.1016/S0304-3959(02)00265-8

Cabral, D. M. C., Bracher, E. S. B., Depintor, J. D. P., \& ElufNeto, J. (2014). Chronic pain prevalence and associated factors in a segment of the population of São Paulo City. The Journal of Pain, 15(11), 1081-1091. https://doi.org/10.1016/j. jpain.2014.07.001

Campbell, L. C., Andrews, N., Scipio, C., Flores, B., Feliu, M. H., $\&$ Keefe, F. J. (2009). Pain coping in Latino populations. The Journal of Pain, 10(10), 1012-1019. https://doi.org/10.1016/j. jpain.2009.03.004

Farias Oliveira Nunes, M., Okino, E. T. K., Noce, M. A., \& Jardim-Maran, M. L. C. (2008). Interesses profissionais: perspectivas teóricas e instrumentos de avaliação. Avaliação Psicológica, 7(3), 403-414.

Ferreira, K. A. S. L., de Siqueira, S. R. D. T., \& Teixeira, M. J. (2008). Atendidos em centro multidisciplinar de dor. Cadernos Saúde Coletiva, 16(3), 449-470.

Flor, H., Elbert, T., Mühlnickel, W., Pantev, C., Wienbruch, C., \& Taub, E. (1998). Cortical reorganization and phantom phenomena in congenital and traumatic upper-extremity amputees. Experimental Brain Research, 119(2), 205-212. https://doi.org/10.1007/s002210050334

Garcia Capitão, C., Scortegagna, S. A., \& Baptista, M. N. (2005). A importância da avaliaçãopsicológica na saúde. Avaliação Psicológica, 4(1).

Goldberg, D. S., \& McGee, S. J. (2011). Pain as a global public health priority. BMC Public Health, 11(1), 770. https://doi. org/10.1186/1471-2458-11-770

Goldman, J., Conrad, D. F., Ley, C., Halperin, D., Sanchez, M. D. L. L., Villacorta, R., \& Parsonnet, J. (2002). Validation of Spanish language dyspepsia questionnaire. Digestive Diseases and Sciences, 47(3), 624-640. https://doi. org/10.1023/A:1017936623783

Jakobsson, U., Klevsgård, R., Westergren, A., \& Hallberg, I. R. (2003). Old people in pain: a comparative study. Journal of pain and symptom management, 26(1), 625-636. https://doi. org/10.1016/S0885-3924(03)00145-3
Keefe, F. J., Rumble, M. E., Scipio, C. D., Giordano, L. A., \& Perri, L. M. (2004). Psychological aspects of persistent pain: current state of the science. The Journal of Pain, 5(4), 195-211. https:// doi.org/10.1016/j.jpain.2004.02.576

Kreling, M. C. G. D., Cruz, D. A. L. M., \& Pimenta, C. A. D. M. (2006). Prevalência de dor crônica em adultos. Revista Brasileira de Enfermagem, 59(4), 509-13. https://doi. org/10.1590/S0034-71672006000400007

Linton, S. J. (2000). A review of psychological risk factors in back and neck pain. Spine, 25(9), 1148-1156.

Loduca, A. (1998). Eu e minha dor: convivendo com um processo crônico. Dissertação de Mestrado, Pontifícia Universidade Católica de São Paulo, São Paulo, SP, Brasil.

Loduca, A. (2007). O tratamento da dor crônica na minha biografia: um estudo sobre a compreensão psicológica da adesão ao tratamento na Clínica de Dor. Tese de doutorado, Pontifícia Universidade Católica de São Paulo, São Paulo, SP, Brasil.

Loduca, A., \& Samuelian, C. (2003). Avaliação psicológica do doente com dor. In: M. J. Teixeira (Ed.). Dor: contexto interdisciplinar. (Vol. 1, pp. 191-204). Editora Maio.

Loduca, A., \& Samuelian, C. (2009). Avaliação psicológica: do convívio com dores crônicas à adesão ao tratamento na clínica de dor. In: O. Alves Neto (Ed.). Dor, princípios e prática. (Vol. 1, pp. 382-97) $1^{\mathrm{a}}$ ed. Artmed.

Loduca, A., Müller, B. M., Amaral, R., Souza, A. C. M. D. S., Focosi, A. S., Samuelian, C., Yeng, L. T., \& Batista, M. (2014). Retrato de dores crônicas: percepção da dor através do olhar dos sofredores. Revista Dor, 15(1), 30-35. https://doi. org/10.5935/1806-0013.20140008

Loduca, A., Müller, B. M., \& Samuelian, C. (2018). Retrato de Dores (1a). Tapsi.

Manek, N. J., \& MacGregor, A. J. (2005). Epidemiology of back disorders: prevalence, risk factors, and prognosis. Current opinion in rheumatology, 17(2), 134-140. https://doi. org/10.1097/01.bor.0000154215.08986.06

McBeth, J., Macfarlane, G. J., Hunt, I. M., \& Silman, A. J. (2001). Risk factors for persistent chronic widespread pain: a community-based study. Rheumatology, 40(1), 95-101. https:// doi.org/10.1093/rheumatology/40.1.95

McWhinney, I. R. (1981). An introduction to family medicine. Oxford University Press. 
Melzack, R. (2001). Pain and the neuromatrix in the brain. Journal of Dental Education, 65(12), 1378-1382. https://doi.org/10.10 02/j.0022-0337.2001.65.12.tb03497.x

Melzack, R. (2005). Evolution of the neuromatrix theory of pain. The Prithvi Raj Lecture: presented at the third World Congress of World Institute of Pain, Barcelona 2004. Pain Practice, 5(2), 85-94. https://doi.org/10.1111/j.1533-2500.2005.05203.x

Melzack, R., \& Wall, P. D. (1967). Pain mechanisms: a new theory. Survey of Anesthesiology, 11(2), 89-90.

Menzel, N. N. (2007). Psychosocial factors in musculoskeletal disorders. Critical care nursing clinics of North America, 19(2), 145-153. https://doi.org/10.1016/j.ccell.2007.02.006

Merskey, H. (1991). The definition of pain. European Psychiatry, 6(4), 153-159. https://doi.org/10.1017/S092493380000256X

Moraes Vieira, É. B. de, Garcia, J. B. S., Silva, A. A. M. da, Araújo, R. L. T. M., \& Jansen, R. C. S. (2012). Prevalence, characteristics, and factors associated with chronic pain with and without neuropathic characteristics in Sao Luis, Brazil. Journal of Pain and Symptom Management, 44(2), 239-251. https://doi.org/10.1016/j.jpainsymman.2011.08.014

O'Brien, T., \& Breivik, H. (2012). The impact of chronic painEuropean patients' perspective over 12 months. Scandinavian Journal of Pain, 3(1), 23-29. https://doi.org/10.1016/j. sjpain.2011.11.004

Ocampo, M. L. S., Arzeno, M. E., Piccolo, E. G. e cols. (2001) O processo psicodiagnóstico e as técnicas projetivas. 10ed. Martins Fontes.

Panek, P. E., Skowronski, J. J., \& Wagner, E. E. (2002). Differences on the projective hand test among chronic pain patients reporting three different pain experiences. Journal of Personality Assessment, 79(2), 235-242. https://doi. org/10.1207/S15327752JPA7902_07
Raja, S. N., Carr, D. B., Cohen, M., Finnerup, N. B., Flor, H., Gibson, S., ... \& Song, X. J. (2020). The revised International Association for the Study of Pain definition of pain: concepts, challenges, and compromises. Pain, 161(9), 1976-1982. https:// doi.org/10.1097/j.pain.0000000000001939

Sá, K. N., Baptista, A. F., Matos, M. A., \& Lessa, Í. (2008). Chronic pain and gender in Salvador population, Brazil. Pain, 139(3), 498-506. https://doi.org/10.1016/j.pain.2008.06.008

Shavers, V. L., Bakos, A., \& Sheppard, V. B. (2010). Race, ethnicity, and pain among the US adult population. Journal of Health Care for the Poor and Underserved, 21(1), 177-220. https:// doi.org/10.1353/hpu.0.0255

Szumita, R. P., Szumita, P. M., \& Just, N. (2010). Understanding and managing patients with chronic pain. Oral and maxillofacial surgery clinics of North America, 22(4), 481-494. https://doi. org/10.1016/j.coms.2010.07.005

Taddeo, P. da S., Gomes, K. W. L., Caprara, A., Gomes, A. M. A., Oliveira, G. C. de, \& Moreira, T. M. M. (2012). Acesso, prática educativa e empoderamento de pacientes com doenças crônicas. Ciência \& Saúde Coletiva, 17(11).

Urnes, J., Johannessen, T., Farup, P. G., Lydersen, S., \& Petersen, H. (2006). Digestive symptoms and their psychosocial impact: validation of a questionnaire. Scandinavian Journal of Gastroenterology, 41(9), 1019-1027. https://doi. org/10.1080/00365520600587402

Villemor-Amaral, A. E. D., \& Pasqualini-Casado, L. (2006). A cientificidade das técnicas projetivas em debate. PsicoUSF, 11(2), 185-193. https://doi.org/10.1590/S141382712006000200007

Wall, P. D., \& Melzack, R. (1999). Textbook of pain. Harcourt Publishers Ltd.

Yeng, L. T., \& Teixeira, M. J. (2004). Tratamento multidisciplinar dos doentes com dor crônica. Prática Hospitalar, 35, 21-24. 